\title{
The new flexible mobile fronthaul: digital or analog, or both?
}

\author{
(Invited Paper)
}

\author{
Nathan J. Gomes, Senior Member, IEEE, Philippos Assimakopoulos, Member, IEEE, Mohamed Kenan Al- \\ Hares, Usman Habib, Student Member, IEEE, Shabnam Noor, Student Member, IEEE \\ Communications Research Group, University of Kent, Canterbury, CT2 7NT, United Kingdom \\ Tel: (44) 1227 823719,e-mail: N.J.Gomes@kent.ac.uk
}

\begin{abstract}
It has become apparent that current fronthaul technology cannot be simply extended to meet the projected demands of $5 \mathrm{G}$ and beyond mobile systems. This current technology, based on the transport of sampled radio waveforms, has been the preferred option, with analog radio over fiber reserved to relatively niche application scenarios. However, for future systems, it is recognised that different functional splits between the central location and the remote units are needed; sampled waveform transport is not scalable to these systems. We propose a flexible fronthaul, therefore, in which both digital and analog transport technologies can coexist. Using practical examples from our work, we describe where these technologies can be used in the future fronthaul.
\end{abstract}

Keywords: fiber-wireless integration, fixed-mobile convergence, fiber fronthaul, Ethernet access networks.

\section{INTRODUCTION}

Fifth Generation (5G) and beyond mobile communication networks must provide high bit-rate services to many users, requiring numerous wireless "access nodes" and shorter radio transmission distances [1]. Centralised- or Cloud-Radio Access Networks have been proposed with the Remote Radio Heads (RRHs) acting as these access nodes, and the centralization of the baseband base station functions offering operational cost savings and opportunities for closer coordination and virtualization of base station functions [2]. For current mobile systems, the transport between the baseband units (BBUs) and RRHs, the fronthaul, has been of sampled radio waveforms (usually using the Common Public Radio Interface, CPRI, industry standard). However, for multi-antenna systems and $5 \mathrm{G}$ bandwidths the bit-rates would become too high. For this reason, recent work has proposed moving some of the baseband functions into the remote unit, so that sampled waveforms are no longer transported [3]-[5]. This can reduce bit-rate requirements towards that of the user data itself. However, the split in functions which were designed to reside in a single physical entity can complicate protocol operation and the timing requirements of the information exchange over this new fronthaul [6]. An alternative is to move even the digital-to-analog conversion functions that resided in the RRH to the central unit, resulting in the transport of the analog radio signals over the fronthaul [7]. These signals are already bandwidth-efficient. Such analog radioover fiber techniques have been used in certain niche applications (e.g. distributed antenna systems in shopping malls, sports complexes) [7] but operators have been reluctant to implement them more widely in their access networks with concerns over the signal degradation.

In this paper, we examine the need to move from the traditional functional split and how this affects the requirements of the fronthaul. In Section 2, we examine the different functional split points that have been proposed. In Section 3, we discuss analog radio over fiber transport for the fronthaul. In Section 4, we suggest that a future fronthaul can accommodate both digital and analog transport, using the strengths of each concept. Conclusions are presented in Section 5.

Table 1. Current and projected bit-rate requirements for sampled waveform transport (*10\% overhead).

\begin{tabular}{|c|c|c|c|}
\hline \multicolumn{2}{|c|}{ Current CPRI/ORI interfaces } & \multicolumn{2}{|c|}{ Projected requirements } \\
\hline Line rate & Example use & Possible use & $\begin{array}{l}\text { Approx. } \\
\text { line rate* }\end{array}$ \\
\hline $614.4 \mathrm{Mb} / \mathrm{s}$ & $\begin{array}{l}10 \mathrm{MHz} \text { LTE channel with 8B10B } \\
\text { coding }\end{array}$ & $1 \mathrm{GHz}$ bandwidth, 1 antenna & $35 \mathrm{~Gb} / \mathrm{s}$ \\
\hline $4.9152 \mathrm{~Gb} / \mathrm{s}$ & $8 \times 10 \mathrm{MHz}$ with $8 \mathrm{~B} 10 \mathrm{~B}$ & $8 \times 100 \mathrm{MHz}$ & $28 \mathrm{~Gb} / \mathrm{s}$ \\
\hline $10.1376 \mathrm{~Gb} / \mathrm{s}$ & $10 \times 20 \mathrm{MHz}$ with 64B66B & $8 \times 500 \mathrm{MHz}$ & $141 \mathrm{~Gb} / \mathrm{s}$ \\
\hline $24.33024 \mathrm{~Gb} / \mathrm{s}$ & $24 \times 20 \mathrm{MHz}$ with 64B66B & $128 \times 500 \mathrm{MHz}$ & $2.25 \mathrm{~Tb} / \mathrm{s}$ \\
\hline
\end{tabular}

\section{RADIO ACCESS NETWORK FUNCTIONAL SPLITS}

Table 1 shows the bit-rate requirements of some example CPRI (and Open Radio Interface, ORI) interfaces [8]; rates 1,58 , and the latest, 10, are shown. The rate 10, for example, allows three carrier aggregation and 8 antenna streams. CPRI/ORI over-sample; for the projected requirements, we have assumed less over-sampling is 
possible, and generally less overhead. For example, for the 8 x $100 \mathrm{MHz}$ transport requirement, simply projecting from current CPRI methods would lead to $49.152 \mathrm{~Gb} / \mathrm{s}$, rather than $28 \mathrm{~Gb} / \mathrm{s}$. The 128 antennas corresponds to a massive MIMO configuration, but without possibilities for sectors, etc. Although compression factors of 2 to 3 have been specified, it is evident that future requirements will still easily reach the order of $\mathrm{Tb} / \mathrm{s}$ per RRH.

Figure 1 shows the main base station functions and some of the split points that have been proposed for the new fronthaul [5], [9]. When the split point is near the pink block at left, representing layer 3 functions, what is to the right of this block and placed in a remote unit, is similar to a micro-base station controlled by a macrobase station. Between this and the current CPRI-type split point, we can see some of the new possible splits being proposed. There is a lot of interest in the baseband physical layer (PHY) splits. At Split III, by transporting symbols for each used subcarrier of the IFFT rather than time domain samples, a several-fold bit-rate reduction can be achieved. The bit-rate is more-or-less constant, however. Moving to the Split II, we do not transport data for unused frequency-time transport blocks - this reduces bit-rate requirements according to the load. In both of these cases, we still send signals for each antenna stream. With Split I, we move the antenna pre-coding to the remote unit and send the pre-coding vectors instead. User data is sent rather than data for each antenna stream, and the reduction in bit-rate is of the order of the number of antennas. Moving the remaining baseband PHY functions to the remote unit reduces the bit-rate as coding and error-correction bits are not sent (although, this is usually only a small overhead). The Split $\mathrm{I}$ is often seen as a useful compromise between bit-rate reduction and retention of complexity in the central unit. It is applicable to both downlink, as shown in Fig.1, and uplink. However, when uplink signals from different remote units have to be jointly processed, this is done for Coordinated Multipoint where users are between radio units, the separate antenna flows must be transported to the central unit - Split III may be necessary. This means that in the same network, different split points may be optimal for different users (those near to a radio antenna, and those who may be between two or more). Equally, we could envisage a network containing the new split points and micro-base stations (higher layer splits) and legacy CPRI equipment.

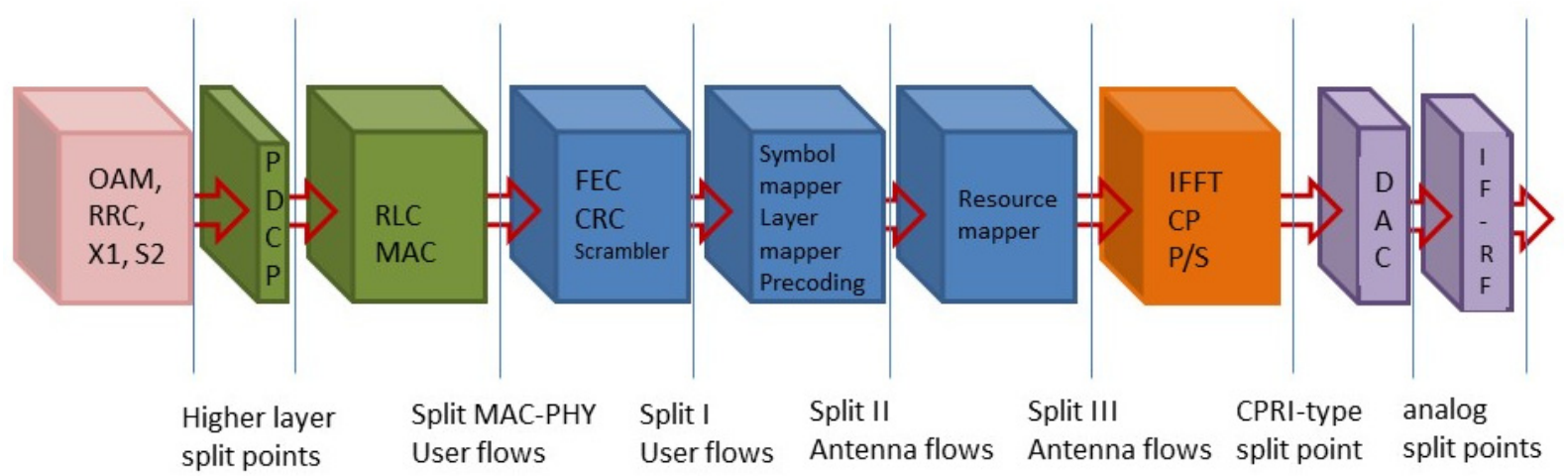

Figure 1. Base station functions and split points

As an example, Table 2 shows the calculated bit-rates in $\mathrm{Gb} / \mathrm{s}$ required for the uplink with different split points and sector loads. Here, the sector is assumed to operate with 8 antennas and $100 \mathrm{MHz}$ bandwidth (comparable to a $49.152 \mathrm{~Gb} / \mathrm{s}$ CPRI requirement or the $28 \mathrm{~Gb} / \mathrm{s}$ projected requirement in Table 1). Ethernet and Radio over Ethernet encapsulation is assumed, as is 6\% control and management traffic and 64B66B overhead.

Table 2. Calculated Ethernet bit-rates (Gb/s) for selected split points at different sector load

\begin{tabular}{l|lll}
\hline \multirow{2}{*}{ Split selection } & \multicolumn{3}{|c}{ Sector load } \\
\cline { 2 - 4 } Split III & $20 \%$ & $50 \%$ & $100 \%$ \\
\hline Split II & 12.2 & 12.2 & 12.2 \\
\hline MAC-PHY & 0.5 & 6.1 & 12.2 \\
\hline \hline
\end{tabular}

The far right of Fig.1 represents components normally located in a RRH. It shows that alternative splits points are also possible by moving functions from there to the central unit and using analog transport (either at IF or $\mathrm{RF}$ ) in the fronthaul. Analog transport is discussed in the following section.

\section{ANALOG RADIO OVER FIBER}

Analog radio over fiber has been of research interest for over 25 years, and commercial products for mobile communications available for some 20 years. However, it has found relatively niche application in fiber-based distributed antenna systems for in-building, shopping mall, sports venue coverage. The problems for I/Q 
sampled waveform transport for new mobile systems has raised the interest in analog transport: in [10] for example, the transport of $14100-\mathrm{MHz}$ channels, closely-separated through the use of the filter-bank multicarrier (FBMC) technique, was demonstrated using only just over $1.5 \mathrm{GHz}$ analog bandwidth - projecting CPRI rates, this would have required over $70 \mathrm{~Gb} / \mathrm{s}$ in a digital fronthaul. In [11], multiple antenna streams and control words are quadrature-amplitude modulated as a $<10 \mathrm{GHz}$ bandwidth analog signal which would be equivalent to a 256 $\mathrm{Gb} / \mathrm{s}$ CPRI stream. While [10] and [11] show the bandwidth efficiency possible using analog techniques, neither demonstrated radio transmission. In [12] a photonic mm-wave up-conversion technique is used for $1 \mathrm{GHz}$ and 3.5 GHz wide OFDM radio signals - from Table 1 it can be seen that these would require bit rates for I/Q sampled waveform transport of the order of $50-150 \mathrm{~Gb} / \mathrm{s}$. A $1 \mathrm{~m}$ radio path was included in the experiment.

In order to discuss the issues involved in analog transport, here, we describe briefly a system for transport of two $1.063 \mathrm{~Gb} / \mathrm{s} 60 \mathrm{GHz}$ signals over a fiber fronthaul. Subcarrier multiplexing of the signals is used, which could be for signals destined for the same remote unit (MIMO signals or signals in different bands) or for signals sent over the fronthaul for different remote units.

In the system, shown in Fig. 2, output from the laser source is split equally with one output modulated by the SCM data and other used to generate an optical carrier $57.8 \mathrm{GHz}$ away using a phase modulator (PM). For this, an RF signal of $28.9 \mathrm{GHz}$ drives the PM and a second order optical sideband is filtered using a dense-wavelength division multiplexing (DWDM) filter. Other setups for mm-wave generation using different modulators and harmonic orders have also been demonstrated. In this experiment, the two SCM channels (16-QAM OFDM) with at $1.9 \mathrm{GHz}$ and $2.5 \mathrm{GHz}$ were generated in an arbitrary waveform generator (AWG) using MATLAB. The resultant signal is amplified using a $17 \mathrm{~dB}$ amplifier and modulates the optical carrier through a Mach-Zehnder modulator (MZM). The output of the MZM is combined with the filtered optical carrier and amplified using an erbium-doped fiber amplifier (EDFA) before transmission over single-mode fiber (SMF). An optical splitter could be used to divide the resultant signal to send it to multiple remote units. At the remote unit, a high bandwidth photodiode $(70 \mathrm{GHz})$ generates the $60 \mathrm{GHz}$ band SCM signal which is amplified and down-converted to IF (in the experiment, this has been done in two stages, simply according to the availability of components). The SCM signal is filtered to obtain the $1.9 \mathrm{GHz}$ and $2.5 \mathrm{GHz}$ signals, which are amplified and captured using a Tektronix Oscilloscope for offline processing.

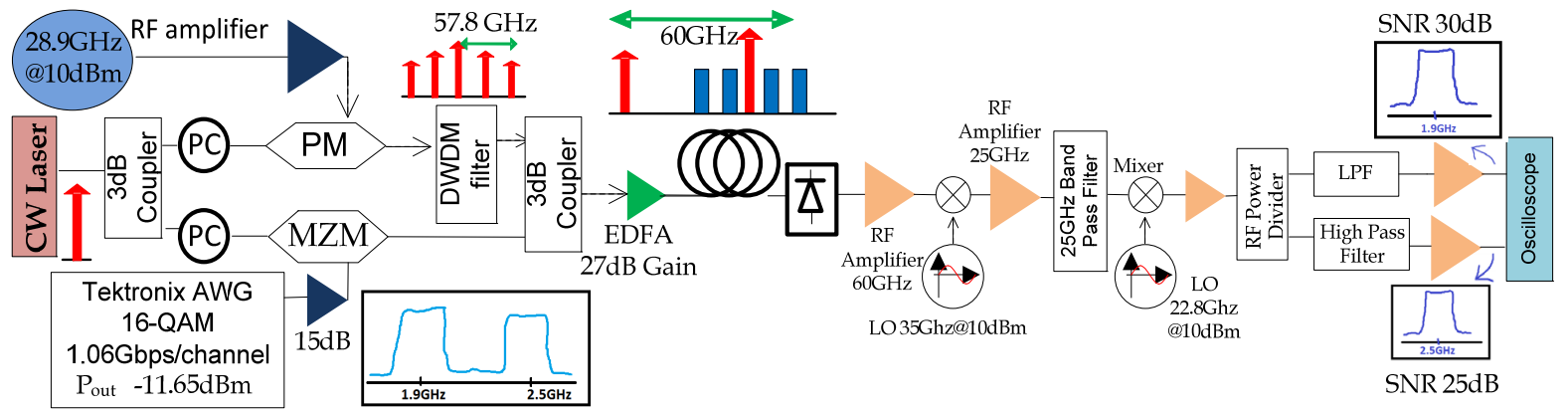

Fig. 2. Photonic generation of $60 \mathrm{GHz}$ mm-wave for SCM data transmission and reception

The suppression ratio achieved for the channels were $25 \mathrm{~dB}$ and $27 \mathrm{~dB}$, respectively. Example results are shown in Fig.3. Figure 3(a) shows the effect of MZM bias on SCM based systems, where an optimum working point between quadrature $(5.6 \mathrm{~V}$ for this modulator) and the null point $(3.1 \mathrm{~V})$ balances between nonlinearity- and noise-limited operation. Figure 3 (b) shows the received constellations when operating near the optimum MZM bias point.
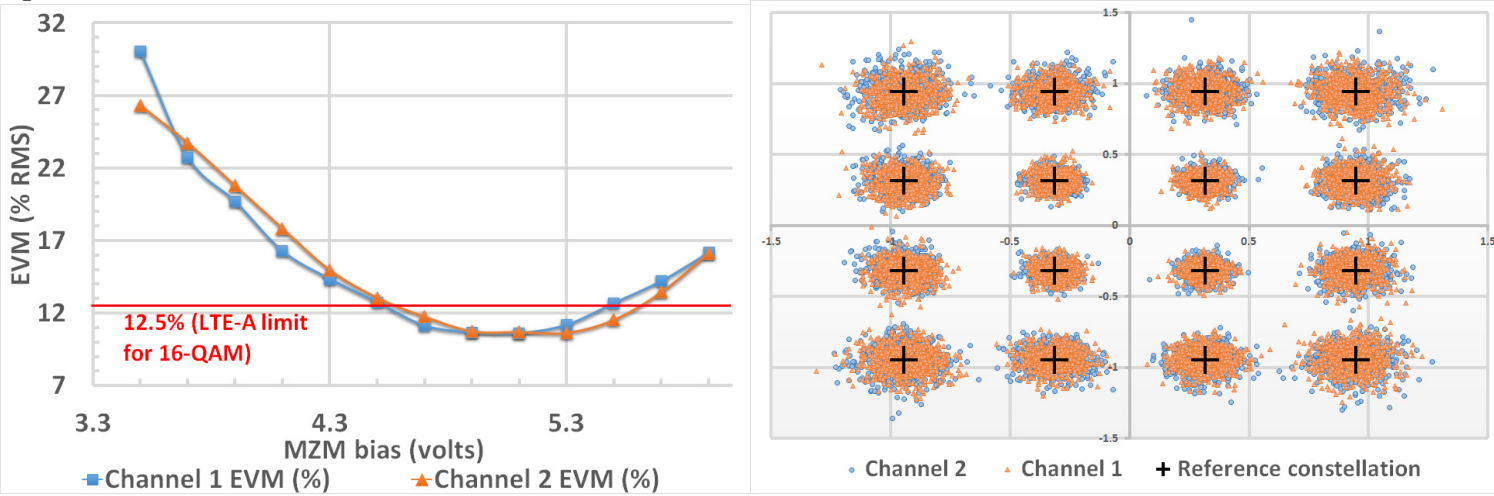

Fig. 3 (a) EVM vs MZM bias (b) received constellations at 4.9 V MZM bias 


\section{FUTURE FRONTHAUL}

Functions can be deployed in different locations in the future fronthaul. Proposals envisage a radio cloud center (RCC), a pool of virtualized base stations carrying out higher layer functions, connecting to remote radio systems, which comprise radio aggregation units (RAUs), with baseband MAC/PHY functions, which can exist physically with the radio parts, or can be separated and connected with CPRI-type links to remote radio units (RRUs) [5]. This architecture is illustrated in Fig.4, but with an important modification: analog links between the RAU and RRUs are used, by as DAC/ADC functions (and possibly RF-IF functions) at the right of Fig.2 are moved into the RAU. This could lead to significant bandwidth savings on the final drop for MIMO signals, and the possibility of using optical beamforming techniques in the analog radio over fiber distribution [13].

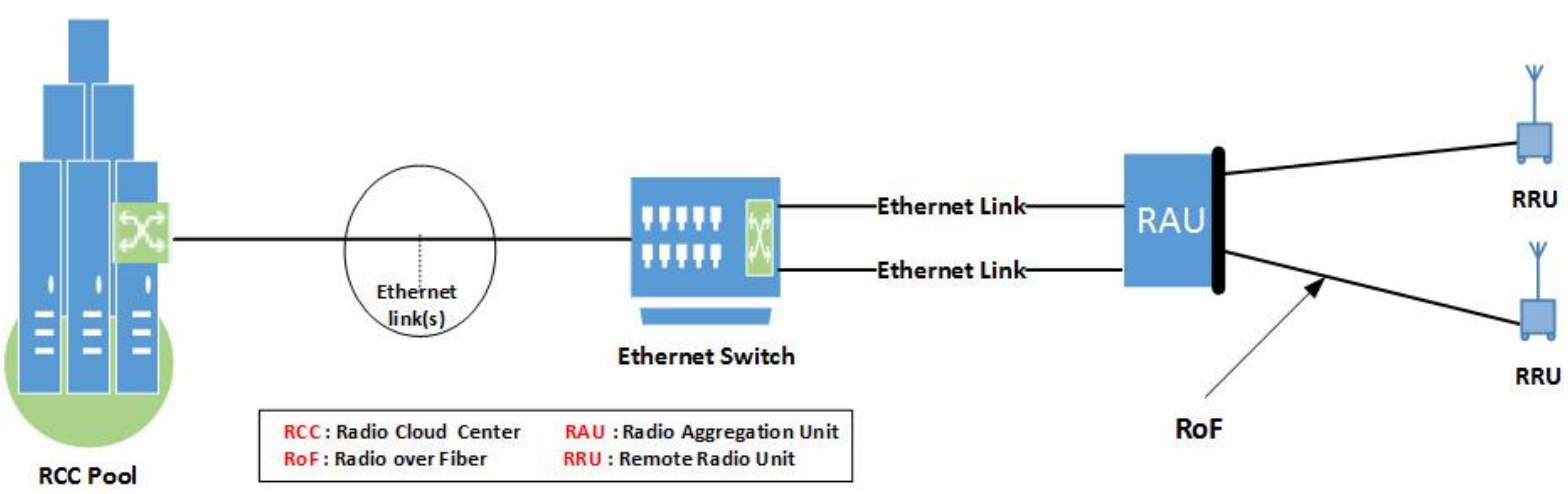

Fig. 4 Fronthaul with digital Ethernet and analog transport

\section{CONCLUSIONS}

The fronthaul is evolving with its functions distributed in order to optimise network performance and user experience. This can lead to both digital and analog solutions being employed.

\section{ACKNOWLEDGEMENTS}

This work has been supported by the European Union's Horizon 2020 programme, contract nos. 644526 and 643297 (iCIRRUS and RAPID), and the UK EPSRC project NIRVANA.

\section{REFERENCES}

[1] NGMN Alliance: NGMN 5G White Paper, Feb. 2015 [available: https://www.ngmn.org/uploads/media/NGMN_5G_White_Paper_V1_0.pdf]

[2] China Mobile Research Institute: C-RAN the road towards green RAN $\bar{N}$, White Paper, v.2.5, Oct. 2011

[3] U. Dötsch, et al:: Quantitative analysis of split base station processing and determination of advantageous features for LTE, Bell Labs Technical J., vol.18, no.1, pp.105-128, Jan. 2013

[4] P. Rost, et al.: Cloud technologies for 5G radio access networks, IEEE Comms Magazine, vol. 52, no.2, pp.68-76, 2014

[5] China Mobile Research Institute, et al: Next generation fronthaul interface, White Paper, v.1.0, Oct. 2015

[6] iCIRRUS: D3.1 Verification of Ethernet as transport protocol for fronthaul/midhaul, Jan. 2016 [available: www.icirrus-5gnet.eu/category/deliverables/]

[7] N.J. Gomes, P. Monteiro, A. Gameiro, eds., Next generation wireless communications using radio over fiber, Wiley, 2012

[8] Ericsson AB, et al.; CPRI specification 6.1, July 2014, [available: www.cpri.info]

[9] NGMN Alliance: Further study on critical C-RAN technologies, March 2015 [available: http://www.ngmn.org/uploads/media/NGMN_RANEV_D2_Further_Study_on_Critical_CRAN_Technologes_v1.0.pdf]

[10] J. Zhang, et al.: Full-duplex, quasi-gapless carrier-aggregation using filter-bank multi-carrier in MMW radio-over-fiber heterogeneous mobile access networks, in Proc. OFC 2016, Anaheim, CA, March 2016, paper Tu2B.2

[11] X. Liu, et al.: CPRI-compatible efficient mobile fronthaul transmission via equalized TDMA achieving $256 \mathrm{~Gb} / \mathrm{s}$ CPRI-equivalent data rate in a single 10-GHz-bandwidth IM-DD channel, in Proc. OFC 2016, Anaheim, CA, March 2016, paper W1H.3

[12] A. Stöhr, et al:: High spectral-efficient 512-QAM-OFDM $60 \mathrm{GHz}$ CRoF system using a coherent photonic mixer (CPX) and an envelope detector, in Proc. OFC 2016, Anaheim, CA, March 2016, paper Tu3B.4

[13] Z. Cao, et al.: Spatial filtering in a broadband in-home OFDM radio-over-fiber network. IEEE Photonic Technol. Letts., vol. 26, no.6, pp. 575-578, March 2014 